\title{
Schema Therapy in adolescents with externalizing behavior problems
}

Citation for published version (APA):

van Wijk - Herbrink, M. F. (2018). Schema Therapy in adolescents with externalizing behavior problems:

Bridging theory and practice. [Doctoral Thesis, Maastricht University]. Uitgeverij BOXPress ||

Proefschriftmaken.nl. https://doi.org/10.26481/dis.20180426mw

Document status and date:

Published: 01/01/2018

DOI:

10.26481/dis.20180426mw

Document Version:

Publisher's PDF, also known as Version of record

\section{Please check the document version of this publication:}

- A submitted manuscript is the version of the article upon submission and before peer-review. There can be important differences between the submitted version and the official published version of record.

People interested in the research are advised to contact the author for the final version of the publication, or visit the DOI to the publisher's website.

- The final author version and the galley proof are versions of the publication after peer review.

- The final published version features the final layout of the paper including the volume, issue and page numbers.

Link to publication

\footnotetext{
General rights rights.

- You may freely distribute the URL identifying the publication in the public portal. please follow below link for the End User Agreement:

www.umlib.nl/taverne-license

Take down policy

If you believe that this document breaches copyright please contact us at:

repository@maastrichtuniversity.nl

providing details and we will investigate your claim.
}

Copyright and moral rights for the publications made accessible in the public portal are retained by the authors and/or other copyright owners and it is a condition of accessing publications that users recognise and abide by the legal requirements associated with these

- Users may download and print one copy of any publication from the public portal for the purpose of private study or research.

- You may not further distribute the material or use it for any profit-making activity or commercial gain

If the publication is distributed under the terms of Article $25 \mathrm{fa}$ of the Dutch Copyright Act, indicated by the "Taverne" license above, 
SUMMARY 
In this dissertation, the theoretical framework and effectiveness of Schema Therapy (ST) is tested in adolescent samples. ST is an effective treatment for adult patients with personality disorders (PDs), and this dissertation is based on the controversial premise that PDs or PD traits, if appropriate, can and should be diagnosed in adolescents. In Chapter 1, the general introduction of this dissertation, scientific support for this premise is discussed. Early diagnosis of PDs or PD traits creates an opportunity for early intervention, for example, using ST. The theoretical framework of ST is presented, discussing the relationships between early maladaptive schemas (EMS), schema coping, and schema modes.

Furthermore, attention is paid to the use of ST principles in (secure) residential treatment, where group care workers face the great challenge of keeping a balance between a flexible therapeutic environment and control. The aims of this dissertation are twofold:

(1) testing the validity of constructs and theoretical basis of ST in adolescents, and

(2) addressing the clinical application and effectiveness of ST with adolescent (in)patients displaying severe externalizing behavior problems, including both individual ST and Safe Path, a milieu-based ST approach.

\section{PART I: ST THEORY IN ADOLESCENTS}

The construct of EMSs has been tested before in adolescents, but research on schema coping and schema modes in adolescents is scarce. Chapter 2 tests the validity of these constructs, and the self-report instruments measuring these constructs, in samples of referred $(n=122)$ and non-referred $(n=577)$ adolescents. Referred adolescents were patients receiving treatment for severe externalizing behavior problems. Results indicated that the same three schema coping styles (surrender, avoidance, and overcompensation) can be distinguished in adolescents as in adults. We also found the same schema modes as previously found in adults. With respect to the higher-order factors of schema modes, we could not confirm two hypothesized models based on theory and previous research. With some exploratory adaptations, we found a good fit for a higher-order model of internalizing, externalizing, overachieving, and healthy modes. Future research should try to replicate this model. This study provides evidence that schema coping and schema modes are valid constructs in adolescents, and that the Schema Coping Inventory and the 80-item Schema Mode Inventory generally are reliable and valid instruments to assess these constructs in adolescent samples.

In Chapter 3, the theoretical framework of ST is tested in adolescents. Previous research in adults has found that schema coping mediates the relationship between specific EMSs and specific schema modes, and we tried to replicate these results in the combined sample of referred and non-referred adolescent from Chapter $2(N=699)$. We also tested a moderating role of schema coping, because from schema theory, it could be hypothesized that the relationships between EMS and schema modes are dependent on the style of schema coping. Furthermore, we tested the combination of such mediating and moderating 
roles of schema coping in moderated mediation models. We found significant mediated effects for all hypothesized relationships. Significant moderated effects were found in half of the hypothesized relationships, and moderated mediation appeared significant in all but one of these relationships. Thus, our findings suggest that schema coping styles are the mechanisms through which EMS activate schema modes, and that sometimes these mechanisms are also influenced by the level of that specific schema coping style. For example, the EMS Abandonment (i.e., the belief that one will always be abandoned by significant others) activates the Abandoned Child mode (i.e., a mode in which one feels abandoned and lost) through a surrendering coping style, and this mechanism is stronger if one tends to use higher levels of surrendering coping. Furthermore, we found that the same EMS can also lead to other schema modes through different coping styles. For example, the EMS Abandonment may also activate a Detached Protector mode (i.e., a mode in which one feels disconnected from others and own needs or feelings) through avoidant coping, and the Angry Child mode (i.e., a mode in which one feels and ventilates uncontrolled anger in response to unmet basic needs) through overcompensatory coping. We also tested higher-order models including relationships between EMSs related to disconnection and rejection experiences (i.e., Abandonment, Mistrust, Defectiveness, Social isolation, and Emotional deprivation), schema coping styles, (surrender vs. overcompensation), schema modes (internalizing vs. externalizing), and behavior problems (internalizing vs. externalizing). We found that the same disconnection and rejection EMSs, through different mechanisms of schema coping and schema modes, may lead to both internalizing and externalizing behavior problems. This suggests that externalizing behavior problems are just as much a manifestation of EMSs related to disconnection and rejection experiences as are internalizing behavior problems. Both adolescents with internalizing (e.g., depression, anxiety) and with externalizing (e.g., oppositional behavior, aggression) problems may possibly benefit from interventions targeting EMSs, schema coping, and schema modes.

In Chapter 4, an experimental study tested the influence of EMSs on the occurrence of negative affect and aggression in response to perceived injustice. Our final sample consisted of 37 boys with severe behavior problems treated in court-mandated, secure residential youth care. In a random selection of these boys, we induced a feeling of unfairness by interviewing them about a recent, personal experience of perceived injustice (experimental condition). The other boys were interviewed about a recent, neutral experience (control condition). We hypothesized that the experimental manipulation would lead to negative affect (anger and/or vulnerable feelings of abandonment and mistrust), which would subsequently lead to aggression, and that this mechanism would be moderated by (i.e., be dependent on the level of) specific EMSs (i.e., Abandonment, Mistrust, Entitlement, and Insufficient Self-control). EMSs and negative affect were measured through self-report, and for vulnerable feelings of abandonment and mistrust we also used an implicit measure. Aggression was measured by a task based on the Taylor Aggression 
Paradigm, distinguishing between unprovoked and provoked (i.e., after hearing sound blasts) aggression. We found that higher levels of the EMS Abandonment increased the effects of perceived injustice on self-reported vulnerable feelings of abandonment and mistrust (no effects were found on implicit vulnerable feelings of mistrust), and that both the EMSs Abandonment and Entitlement increased the effects of perceived injustice on self-reported anger. Furthermore, higher levels of the EMSs Abandonment and Entitlement strengthened the effects of perceived injustice on anger and subsequent unprovoked aggression (no effects were found on provoked aggression). The results suggest that even a vulnerable EMS, such as Abandonment, which is usually associated with internalizing problems, may underlie anger and aggression. These results support the findings in Chapter 3 that interventions targeting EMSs, such as ST, may help ameliorate adolescents' externalizing behavior problems, including aggression.

\section{PART II: EFFECTIVENESS AND CLINICAL APPLICATION OF ST IN ADOLESCENTS WITH EXTERNALIZING BEHAVIOR PROBLEMS}

Chapter 5 includes a multiple case study of four patients with PD traits and severe disruptive behaviors receiving ST in court-mandated, secure residential youth care. We developed an ST treatment protocol for this population, which includes at least 40 individual ST sessions, an ST approach by group care workers, and several ST family sessions. The chapter includes both qualitative and quantitative results of the ST treatment. The qualitative results comprise case narratives describing the techniques we used, working on treatment goals for each patient. The quantitative results comprise tests of changes in behavior problems (reported by patient and by mentor group care worker), self-reported EMSs, and self-reported schema modes. The results support the feasibility of delivering ST in a secure residential treatment center: The therapist was able to use the full range of ST techniques (i.e., working in the here and now with schema modes, experiential techniques, and cognitive-behavioral techniques) with all four patients. We trained and coached treatment teams in ST theory and treatment approach, and were partially successful in involving parents in the ST treatment. We found that all four patients improved in their behavior problems, either reported by the patient or by the mentor care worker, and that most patients also showed significant improvements in schema modes and EMSs. This is the first study providing preliminary evidence for the feasibility and effectiveness of ST for adolescents with PD traits and disruptive behaviors.

Encouraged by the results of the multiple case study, we designed a quasirandomized, controlled trial comparing ST treatment with treatment as usual (TAU) for patients with PD traits and disruptive behaviors in court-mandated, secure residential youth care. The study protocol is described in Chapter 6. We aim to include 128 adolescent patients who: (a) are 14 years or older, (b) are expected to stay in secure residential treatment for at least six months, (c) have at least five PD traits, (d) have an IQ above 70 
and are not referred to a special treatment unit for mentally disabled patients, and (e) are not suffering from acute psychosis or severe autism. Practical issues prevent us from using randomization, so we use quasi-randomization. An independent committee allocates patients to the TAU or ST conditions based on availability of beds and opening slots in caseloads of ST therapists. TAU comprises a therapeutic environment and additional psychotherapy or training based on cognitive-behavioral theory. ST comprises a therapeutic environment in which ST principles are also integrated, and additional individual ST sessions on a twice-per-week basis. At baseline and every three months during treatment, patient's primary group care worker (mentor) completes a checklist on patient's behavior problems (primary outcome). Patients complete self-report instruments on behavior problems, ST-related constructs, quality of life, and group climate (secondary outcomes) at these time points and additionally at three and 12 months after discharge. We expect patients in ST to show greater improvements in outcomes over time, and to better maintain these gains at follow-up than patients in TAU. We also expect ST to be superior with respect to dropout, no shows for individual therapy sessions, and group climate. This chapter describes the analyses we will use for these data (mixed regression analyses), and discusses the relevance of this study, along with its strengths and limitations.Although we aimed to finish this study within three years, we encountered some setbacks that slowed down the rate of patient inclusion. After three years of running this study, we included 69 patients. With this rate of inclusion, we would have to continue data collection for the next 2,5 years to achieve a sample size of 128 patients.

Chapter 7 includes the preliminary results of Safe Path, a milieu-based ST approach, on incidents of aggression (primary outcome), group climate, staff's severe interventions, and staff's team functioning. Over a period of six months, incidents of aggression and severe interventions (i.e., physical interventions, seclusion, and transfer to a more restrictive unit) were coded from daily reports. At a two-month interval, group climate was rated by patients, and team functioning was rated by group care workers. We hypothesized that Safe Path units would show higher or faster improvements in these outcome measures over time (condition * time interaction) than treatment units providing care as usual (CAU; based on cognitive-behavioral principles). The chapter incorporates the aims and content of the Safe Path intervention, and investigates staff's use of the schema mode language (which is one of the main goals of Safe Path) four months after implementation. We found that the implementation process was far from complete (staff from the Safe Path units used the schema mode language in only $9 \%-11 \%$ of the daily reports after two to four months), which could account for the absence of significant interaction effects in our study. We conclude that it is premature to draw conclusions on the effects of Safe Path. A complete analysis of outcomes will be conducted one year after implementation. The preliminary report in this chapter includes examples of Safe Path interventions, and discusses issues arising in the Safe Path coaching. 
Chapter 8 is a Dutch practical manual addressing the application of ST to adolescents with externalizing behavior problems and PD traits. It distinguishes four phases of treatment: a motivation phase, a diagnostic phase, a phase of change, and a phase of generalization, and it places special emphasis on handling schema modes associated with anger (e.g., Angry Child, Angry Protector, Bully and Attack modes). The chapter offers many illustrations of ST techniques, which could support clinicians using ST with this complex population. The chapter also discusses the involvement of parents in the ST treatment, and how to create a ST-based therapeutic environment in residential youth care.

\section{DISCUSSION AND CONCLUSION}

In Chapter 9, the results of all studies in this dissertation are briefly summarized and integrated in a general discussion. The findings support the validity of ST theory in adolescents, and suggest (with preliminary evidence) that adolescent patients with externalizing problems may benefit from ST. This chapter includes methodological considerations and future directions for further research. 
